\title{
Cardia carcinomas of intestinal type are associated with histologic changes in the gastric mucosa
}

\author{
Carlos A. Rubio ${ }^{1}$, Yo Kato ${ }^{2}$, Akio Yanagisawa ${ }^{2}$, Yasuo Ninomiya ${ }^{2}$, and Koichi Mandai ${ }^{3}$ \\ ${ }^{1}$ Department of Pathology, Karolinska Institute, 17176 Stockholm, Sweden \\ ${ }^{2}$ Cancer Institute, Tokyo, Japan \\ ${ }^{3}$ National Shikoku Cancer Center Hospital, Matsuyama, Japan
}

\begin{abstract}
:
Background. Previous studies of Japanese patients showed that distal gastric carcinomas (of the corpus or the antrum) were associated with histologic changes in the non-neoplastic gastric mucosa. These changes (cells with ciliated metaplasia, with large and small mucus-negative vacuoles, intramucosal glandular cysts, and extensive intestinal metaplasia) were often found in carcinomas of intestinal type.

Methods. In the present work, similar mucosal changes were searched for in surgical specimens carrying a carcinoma of the cardia. A total of 12079 archival histologic sections, corresponding to 563 gastrectomies performed in Japanese patients, were analyzed.

Results. Of the specimens with cardia carcinoma of intestinal type seen in Tokyo $(n=169)$ and in Matsuyama $(n=168)$, $53.3 \%$ and $63.1 \%$ contained intramucosal cysts; $39.6 \%$ and $\mathbf{5 1 . 8} \%$, ciliated metaplastic cells; $34.9 \%$ and $30.4 \%$, large vacuolated cells; and $7.7 \%$ and $11.9 \%$, small vacuolated cells; and $69.8 \%$ and $68.5 \%$ had high intestinal metaplasia scores, respectively. These percentages were significantly higher than those for cardia carcinomas of diffuse type in Tokyo $(n=153)$ and in Matsuyama $(n=73)$, in which $30.7 \%$ and 34.2\% of the specimens had intramucosal cysts; $17.6 \%$ and $17.8 \%$, ciliated metaplastic cells; $14.4 \%$ and $9.6 \%$, large vacuolated cells; and $3.9 \%$ and $4.1 \%$, small vacuolated cells; and $43.1 \%$ and $39.7 \%$ had high intestinal metaplasia scores, respectively.

Conclusion. Thus, similarly to carcinomas of the corpus or the antrum, cardia carcinomas of intestinal type are associated with histologic changes in the gastric mucosa. The possibility that these changes are evoked by environmental factors was entertained.
\end{abstract}

Key words: cardia carcinoma, environmental factors, nonneoplastic changes

\section{Introduction}

Gastric carcinoma continues to dominate as a leading cause of cancer-related death in Japan and other Asian countries [1,2]. In the West, however, the incidence of gastric carcinoma has been declining in past decades, more notably for carcinomas located in the gastric corpus and in the antrum, whereas a rapid increase in incidence has been recorded for carcinomas located in the cardia [3]. Although the causes for this increase remain elusive, it has been suggested that cardia cancer may evolve through an etiologic pathway that is different from that for distal gastric carcinomas. In fact, cardia tumors differ from carcinomas of the corpus and antrum in terms of sex, race, and prognosis [3].

Previous studies in Japanese gastrectomy specimens having carcinomas of the corpus or the antrum (ie, distal stomach) showed that the non-neoplastic gastric mucosa contained intramucosal cysts [4] and pyloric cells with ciliated metaplasia [5], with large or small mucus-negative vacuoles [6], with glassy cytoplasm [7], or with atypical mitoses [8]; further, there was extensive intestinal metaplasia (IM) [9]. These non-neoplastic changes were more often found in gastric specimens having a carcinoma of intestinal type [10-15], a tumor believed to evolve by the influence of environmental carcinogens [1-3]. The possibility that these nonneoplastic changes could also evolve as a result of a similar environmental influence was entertained.

The aim of the present work was to investigate whether the aforementioned non-neoplastic mucosal changes could also occur in specimens having a cardia carcinoma.

\section{Material and methods}

Histological sections from 563 gastrectomy specimens with cardia carcinomas filed at the respective hospitals
Offprint requests to: C.A. Rubio

Received on July 21, 1999; accepted on Jan. 5, 2000 
during a 10-year period were reviewed. Sections had been stained with hematoxylin and eosin.

Localization of the carcinoma in the cardia was established on the basis of the clinical data and of gross examination of the resected stomachs.

\section{Definitions}

Cardia. Cardia carcinomas were defined according to the classification proposed by Siewert and recently approved during the consensus conference held during the second International Gastric Cancer Congress in Munich [16].

Type I. Cardia carcinoma extending to the esophagus with the center lying $1-5 \mathrm{~cm}$ above the anatomic esophago-gastric junction.

Type II. Cardia carcinoma extending to the stomach with the center located within $1 \mathrm{~cm}$ above and $2 \mathrm{~cm}$ below the anatomic esophago-gastric junction.

Type III. Cardia carcinoma extending to the stomach with the center lying within $2-5 \mathrm{~cm}$ below the anatomic esophago-gastric junction.

Included in the present work were cardia carcinomas type II and type III of the Munich Classification [16].

\section{Assessment of the non-neoplastic associated changes}

The occurrence of intramucosal cysts and intestinal metaplasia (IM) was assessed at low-power examination $(4 \times$ objective) and the detection of cells with ciliated metaplasia, with large or small (mucus-negative) vacuoles, and/or with glassy cytoplasm was assessed at high-power magnification $(40 \times)$.

The presence of intestinal metaplasia (IM) was noted in each section. IM was classified (using low-power examination) as low-score IM or high-score IM, as reported elsewhere $[15,18,19]$. Briefly, specimens with low-score IM were those having individual foci (ie, "spots") of IM in at least one of the sections. Specimens with high-score IM were those showing one or more entire field(s) of IM in at least one of the sections.

IM was considered widespread in those specimens having IM in $50 \%$ or more of the sections (irrespective of whether the IM score was high or low).

Carcinomas were histologically classified, according to Laurén and Nevalainen [20], as intestinal and diffuse types. Carcinomas of mixed histologic type were classified according to the predominant histologic subtype.

Results were analyzed by the $\chi^{2}$ test and by the Mann-Whitney $U$ non-parametric test.

\section{Results}

Of the 322 specimens seen in Tokyo, $52.5 \%(n=169)$ had intestinal type carcinomas and the remaining $47.5 \%$ $(n=132)$, diffuse type carcinomas. Of the 241 specimens seen in Matsuyama, $69.7 \%(n=168)$ had an intestinal type carcinoma and $30.3 \%(n=73)$ a diffuse type carcinoma.

Age. The age distribution of the patients is shown in Table 1. Of the cases with intestinal carcinoma seen in Tokyo, 34.3\% (58/169) occurred in patients 70 years of age or older, the rate in Matsuyama being $32.7 \%$ $(55 / 168)$.

Of the cases with diffuse carcinoma, $14.4 \%(22 / 153)$ of those seen in Tokyo were in patients 70 years of age or older, the rate in Matsuyama being $13.7 \%$ (10/73).

Sex. Of the cases with intestinal carcinoma seen in Tokyo, $55.6 \%(85 / 153)$ of the patients were men and for those seen in Matsuyama, 85.7\% (144/168) were men. Of the cases with diffuse carcinoma seen in Tokyo, 55.5\% (85/153) of the patients were men, and for those reviewed in Matsuyama, 56.2\% (41/73) were men.

Number of sections. A total of 12079 sections were reviewed. In Tokyo, a total of 6531 sections, corresponding to the 322 gastrectomy specimens, were reviewed. The mean number of sections per gastrectomy was 20.3 (range, 5-162 sections).

In Matsuyama, a total of 5548 sections, corresponding to the 241 gastrectomy specimens, were reviewed. The mean number of sections/gastrectomy was 23.0 (range, 5-73 sections).

\section{Non-neoplastic mucosal changes in gastrectomy} specimens (Tables 2 and 3)

Intramucosal cysts. In specimens with cardia carcinomas of intestinal type, intramucosal cysts (Fig. 1) were found in 53.3\% (90/169) of those seen in Tokyo and in $63.1 \%$ $(106 / 168)$ of those seen in Matsuyama.

In specimens with cardia carcinomas of diffuse type, intramucosal cysts were found in $30.7 \%$ (47/153) of the specimens seen in Tokyo and in $34.2 \%$ (25/73) of those seen in Matsuyama.

Table 1. Age distribution of 563 patients who had gastrectomy for cardia carcinoma

\begin{tabular}{lrc}
\hline Age $($ years $)$ & Tokyo & Matsuyama \\
\hline$\leq 29$ & $2(0.6 \%)$ & $1(0.4 \%)$ \\
$30-39$ & $16(4.9 \%)$ & $7(2.9 \%)$ \\
$40-49$ & $38(11.8 \%)$ & $28(11.6 \%)$ \\
$50-59$ & $86(26.7 \%)$ & $51(21.1 \%)$ \\
$60-69$ & $100(31.1 \%)$ & $92(38.2 \%)$ \\
$70-79$ & $70(21.7 \%)$ & $58(24.0 \%)$ \\
$\geq 80$ & $10(3.1 \%)$ & $7(2.9 \%)$ \\
Total & 322 & 241 \\
\hline
\end{tabular}


Table 2. Non-neoplastic changes in the gastric mucosa in specimens from gastrectomies with cardia carcinoma of intestinal type

\begin{tabular}{lcr}
\hline Histology & Tokyo $(n=169)$ & $\begin{array}{r}\text { Matsuyama } \\
(n=168)\end{array}$ \\
\hline Intramucosal cysts & $90(53.3 \%)$ & $106(63.1 \%)$ \\
Ciliated metaplasia & $67(39.6 \%)$ & $87(51.8 \%)$ \\
Large vacuolated cells & $59(34.9 \%)$ & $51(30.4 \%)$ \\
Small vacuolated cells & $13(7.7 \%)$ & $20(11.9 \%)$ \\
Glassy cells & $4(2.4 \%)$ & $2(1.2 \%)$ \\
High IM score & $118(69.8 \%)$ & $115(68.5 \%)$
\end{tabular}

IM, Intestinal metaplasia (see text for explanation of score)

Table 3. Non-neoplastic changes in the gastric mucosa in specimens from gastrectomies with cardia carcinoma of diffuse type

\begin{tabular}{lcc}
\hline Histology & Tokyo $(n=153)$ & $\begin{array}{c}\text { Matsuyama } \\
(n=73)\end{array}$ \\
\hline Intramucosal cysts & $47(30.7 \%)$ & $25(34.2 \%)$ \\
Ciliated metaplasia & $27(17.6 \%)$ & $13(17.8 \%)$ \\
Large vacuolated cells & $22(14.4 \%)$ & $7(9.6 \%)$ \\
Small vacuolated cells & $6(3.9 \%)$ & $3(4.1 \%)$ \\
Glassy cells & $4(2.6 \%)$ & $1(1.4 \%)$ \\
High IM score & $66(43.1 \%)$ & $29(39.7 \%)$ \\
\hline
\end{tabular}

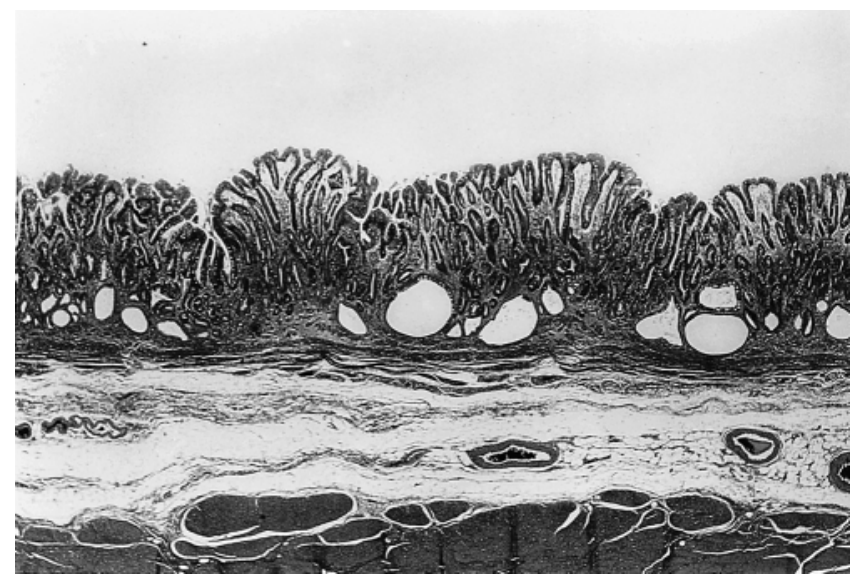

Fig. 1. Gastric mucosa showing intramucosal cysts. $H \& E$, $\times 10$

Cells with ciliated metaplasia. In specimens with cardia carcinomas of intestinal type, ciliated metaplastic cells (Fig. 2) were found in $39.6 \%(67 / 169)$ of the specimens seen in Tokyo and in 51.8\% (87/168) of those seen in Matsuyama.

In specimens with cardia carcinomas of diffuse type, ciliated metaplastic cells were recorded in $17.6 \%(27 /$ $153)$ of the specimens in Tokyo and in $17.8 \%(13 / 73)$ in Matsuyama.

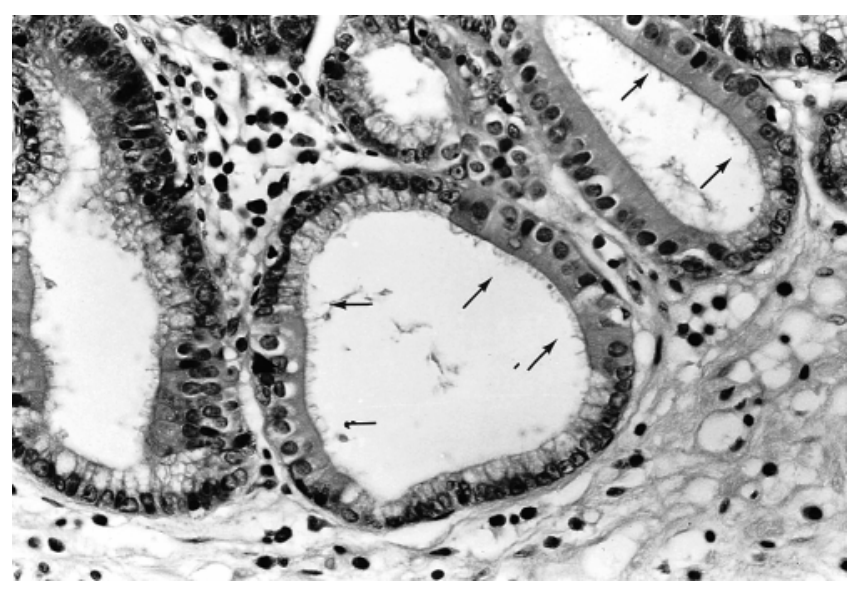

Fig. 2. High-power view of the gastric mucosa, showing ciliated metaplastic cells (arrows). Grimelius, $\times 100$

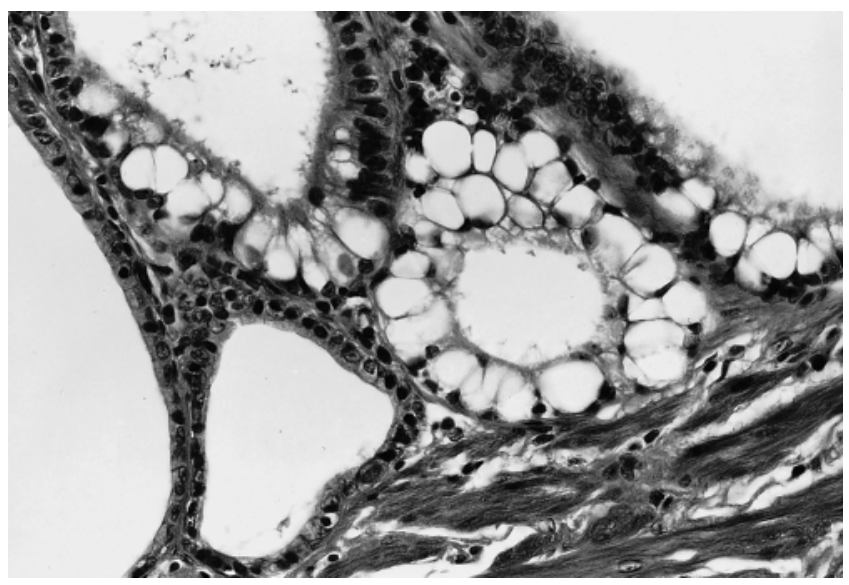

Fig. 3. Gastric mucosa with large, mucus-negative vacuoles in pyloric cells. $\mathrm{H} \& \mathrm{E}, \times 100$

Cells with large vacuoles. Large vacuolated cells (Fig. 3) were found in specimens with cardia carcinomas of intestinal type in $34.9 \%(59 / 169)$ of those seen in Tokyo and in 30.4\% (51/168) of those in Matsuyama.

In specimens with cardia carcinomas of diffuse type, cells with large vacuoles were recorded in $14.4 \%$ (22/ $153)$ of the specimens seen in Tokyo and in $9.6 \%$ (7/73) of those seen in Matsuyama.

Cells with small vacuoles. In specimens with cardia carcinomas of intestinal type, small vacuolated cells (Fig. 4) were found in $7.7 \%(13 / 169)$ of those seen in Tokyo and in $11.9 \%(20 / 168)$ of those from Matsuyama.

In specimens with cardia carcinomas of diffuse type, cells with small vacuoles were recorded in $3.9 \%(6 / 153)$ of the specimens seen in Tokyo and in 4.1\% (3/73) of those seen in Matsuyama. 


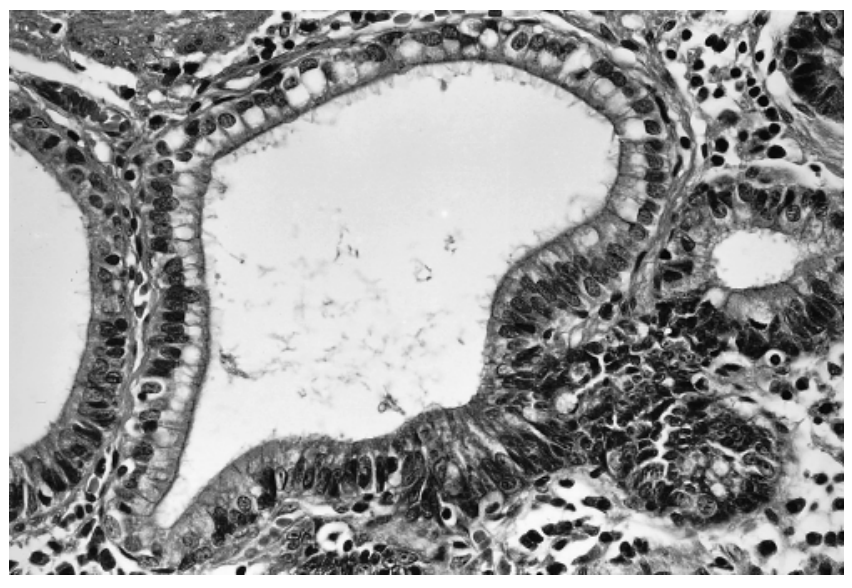

Fig. 4. Gastric mucosa with small, mucus-negative vacuoles in the subnuclear aspect of pyloric cells. $H \& E, \times 25$

Cells with glassy cytoplasm. This type of pyloric cell was characterized by a homogeneous, non-vacuolated, cytoplasm, and with a relatively large nucleous located at the lateral or at the luminal aspect of the cells (Fig. $5)$.

Cells with glassy cytoplasm were recorded in $2.5 \%(8 /$ $322)$ of the Tokyo specimens, and in $1.2 \%$ (3/241) of the Matsuyama specimens.

In specimens with cardia carcinomas of intestinal type, cells with glassy cytoplasm were recorded in $2.4 \%(4 / 169)$ in Tokyo and in $1.2 \%(2 / 168)$ in Matsuyama.

In specimens with cardia carcinomas of diffuse type, cells with glassy cytoplasm were recorded in $2.6 \%$ (4/ $153)$ in Tokyo and in 1.4\% (1/73) in Matsuyama.

Specimens with high IM score. High IM scores were found in $57.1 \%(184 / 322)$ of the specimens reviewed in Tokyo, and in 59.8\% (144/241) of those seen in Matsuyama.

In specimens with cardia carcinomas of intestinal type, high IM scores were found in $69.8 \%$ (118/169) of the cases reviewed in Tokyo and in $68.5 \%$ (115/168) of the cases seen in Matsuyama.

In Tokyo specimens with cardia carcinomas of diffuse type, high IM scores were recorded in 43.1\% (66/153); and in those from Matsuyama in 39.7\% (29/73).

Specimens with extensive IM. Extensive IM (50\% or more of the gastrectomy/sections with IM) was recorded in $70.5 \%(227 / 322)$ of the specimens seen in Tokyo and in $67.2 \%(162 / 241)$ of those investigated in Matsuyama (Fig. 6).

Localization of the non-neoplastic changes. Cells with ciliated metaplasia, with large or small mucus-negative vacuoles, or with glassy cytoplasm were found in the mucosa of the antrum, and less frequently in the mucosa of the corpus. Similar cells were not recorded in the

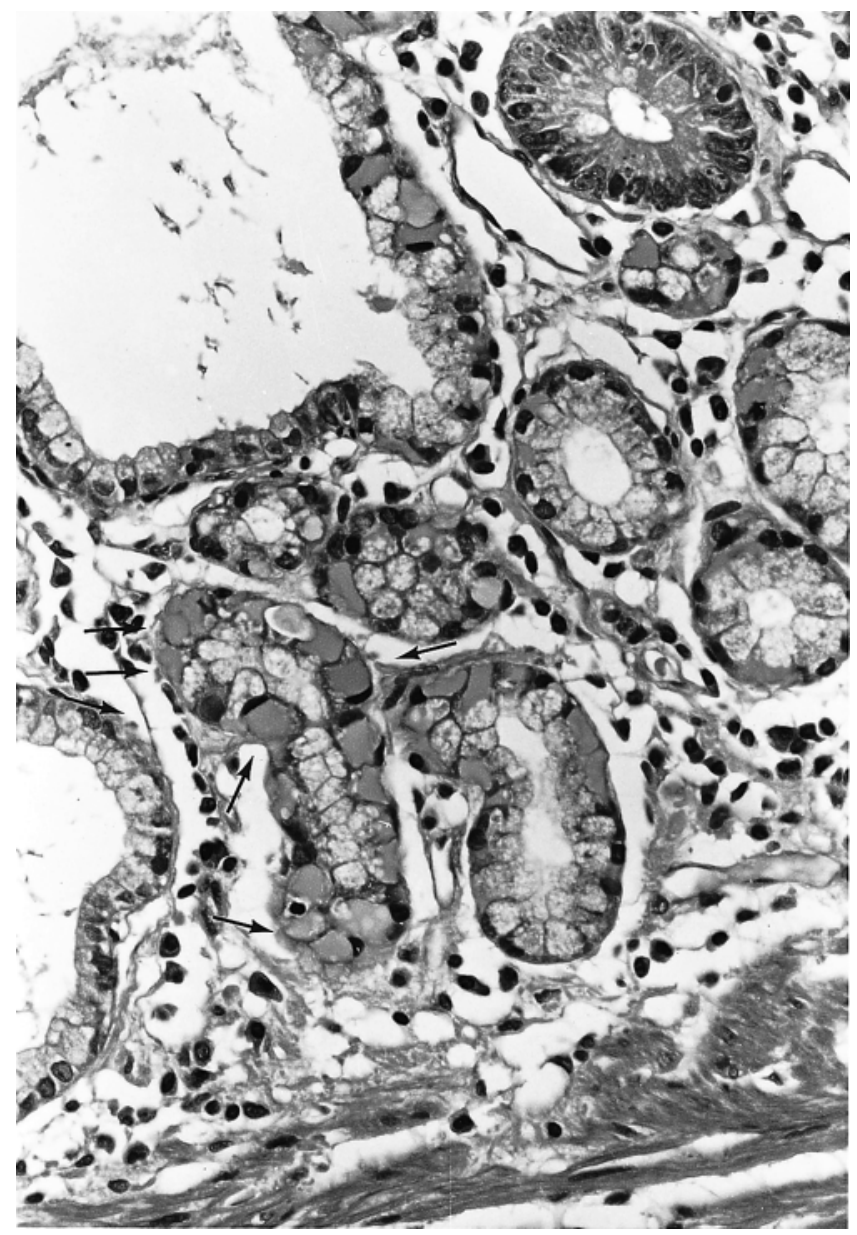

Fig. 5. Gastric mucosa with pyloric cells having glassy cytoplasm (arrows). H\&E, $\times 25$

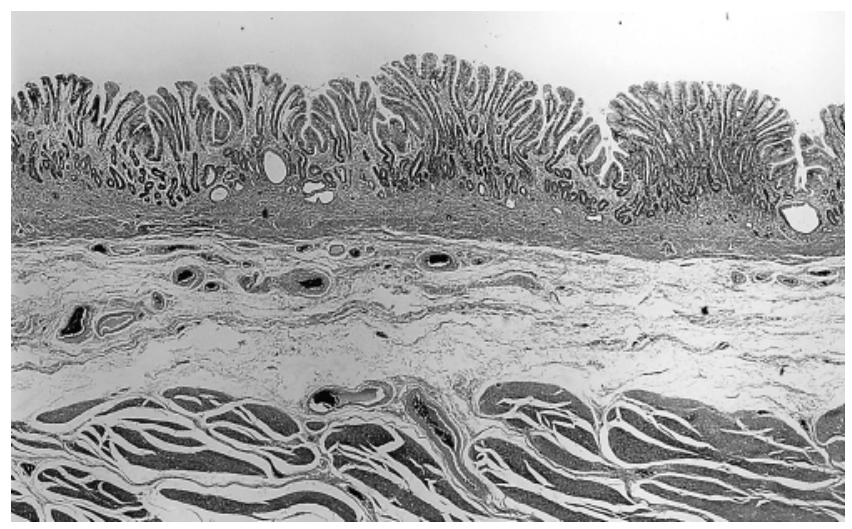

Fig. 6. Gastric mucosa showing extensive intestinal metaplasia occupying the entire field at low-power examination. $\mathrm{H} \& \mathrm{E}, \times 25$

non-neoplastic gastric mucosa in zone A or in the juxtaposed zone B.

Intramucosal cysts were found in sections from the entire stomach, particularly in areas with extensive IM. 
Of the 322 cardia carcinomas seen in Tokyo, 48 $(14.9 \%)$ were regarded as type II, and $29.2 \%$ of these $(14 / 48)$ were immediately surrounded by IM, as IM was present in all sections (including those containing part of the tumor).

Of the 241 cardia carcinomas seen in Matsuyama, 23 tumors $(9.5 \%)$ were regarded as type II, and $26.1 \%$ of these $(6 / 23)$ were immediately surrounded by IM, as IM was present in all sections (including those containing part of the tumor).

\section{Discussion}

In this investigation, 563 gastrectomy specimens from Japanese patients carrying type II or type III cardia carcinoma [16] were reviewed. Results demonstrated that cardia carcinomas were associated with nonneoplastic histologic changes elsewhere in the stomach.

Specimens carrying cardia carcinomas of intestinal type more often showed intramucosal glandular cysts, cells with ciliated metaplasia, cells with large or small mucus-negative vacuoles, and/or extended IM than specimens carrying cardia carcinomas of diffuse type.

Intramucosal cysts and ciliated metaplasia were usually found in specimens having intestinal metaplasia. However, not all specimens containing intramucosal cysts had ciliated metaplasia, suggesting that these cysts may not be the only underlying cause of the appearance of ciliated metaplastic cells in the gastric mucosa.

The percentage of cells with glassy cytoplasm was similarly low in the two Japanese cities. These cells have also been found in specimens without gastric carcinoma, not only in humans [7,21] but also in non-human primates [22]. The "glassy" material found in the cytoplasm of these cells contains Cytasin C (normally excreted by pyloric cells [23]). No difference was found in the occurrence of these cells in the different histologic types of tumor.

In the present work, Helicobacter pylori was not searched for, because this study encompassed the late stages of gastric diseases, in which the organism's presence is known to be influenced by several parameters, such as the washing of the specimen after surgery, and the presence of intestinal metaplasia, mucosal atrophy, and hypoacidity (the latter three often being present in specimens with gastric carcinoma). Moreover, special stains for detecting Helicobacter pylori (difficult to demonstrate in hematoxylin-eosin-stained sections when only a few organisms are present) were not available. On the other hand, gastric specimens from Swedish patients without carcinoma showed - in the presence of Helicobacter pylori detected by special stains [24,25] - absence of intramucosal cysts, and absence of cells with ciliated metaplasia, glassy cytoplasm, and large or small mucus-negative vacuoles. These findings strongly suggest that the cause of the occurrence of the aforementioned mucosal changes may not be related to the presence of Helicobacter pylori in the stomach.

According to the present concept of carcinogenesis, several alternative genetic pathways may be needed for the conversion of normal epithelial cells to clinical cancer. These recognized steps are the accumulation of multiple mutations affecting DNA repair genes, oncogenes, and tumor suppressor genes. Whether the non-neoplastic changes in the gastric mucosa are also evoked by the same or by other genetic mutants remains unknown.

In conclusion, non-neoplastic mucosal changes are present in cardia carcinomas (types II and III) of Japanese patients, more notably in carcinomas of intestinal phenotype than in those of diffuse phenotype. Similar results have been reported earlier for carcinomas of intestinal type in the corpus and the antrum in Japanese patients $[4-9,17,18]$, indicating that nonneoplastic histologic changes usually evolve in association with a particular histologic type, irrespective of the location of the tumor within that organ.

Although cardia carcinomas differ from distal carcinomas in terms of sex, race, and prognosis [3], the mucosal "soil" on which gastric carcinomas of intestinal type develop may be similar for the entire organ.

Acknowledgments The study was supported by the Japan Society for the Promotion of Science, The Swedish Academy of Sciences, and The Karolinska Institute and Hospital.

\section{References}

1. Ming SC. Cellular and molecular pathology of gastric carcinoma and precursor lesion: a critical review. Gastric Cancer 1998;1:3150.

2. Neugut AI, Hayek M, Howe G. Epidemiology of gastric cancer. Semin Oncol 1996;23:281-91.

3. Lagergren J. Cancer of the esophagus and gastric cardia: etiological aspects. Thesis. Stockholm: Repro-Print AB; 1999.

4. Rubio CA, Kato Y, Sugano H, Hirota T. The intramucosal cysts of the stomach. VII: a pathway of gastric carcinogenesis. J Surg Oncol 1986;32:214-9.

5. Rubio CA, Kato Y. Ciliated metaplasia in the gastric mucosa. Jpn J Cancer Res 1986;77:282-6.

6. Rubio CA, Kato Y. Classification of vacuolated cells in the gastric mucosa. J Surg Oncol 1987;34:128-32.

7. Rubio CA. Five types of pyloric cells in the antral mucosa of the stomach Pathol Res Pract 1992;188:157-61.

8. Rubio CA, Kato Y, Kitagawa T. Frequency of atypical mitoses in gastric intestinal metaplasia in Japanese patients. Jpn J Cancer Res 1994;85:284-9.

9. Rubio CA, Matthies M, Itabashi M, Hirota T. Image quantitation of intestinal metaplasia in entire gastrectomy specimens from Swedish and Japanese patients. Jpn J Cancer Res 1996;87:6628. 
10. Rubio CA, Jass JR, King A. Gastric cell phenotypes and intestinal metaplasia in Polynesian and non-Polynesian residents of New Zealand. J Environ Pathol Toxicol Oncol 1994;13:243-9.

11. Rubio CA, Pisano R, Llorens P, Duarte I. A comparative study between the gastric mucosa of Chileans and other dwellers of the Pacific Basin. Jpn J Cancer Res 1996;87:117-21.

12. Rubio CA, Namiki H, Stemmermann G. Geographic variations in the histologic characteristics of the gastric mucosa. J Environ Pathol Toxicol Oncol 1993;12:89-92.

13. Rubio CA, Stemmermann G, Hayashi T. Ciliated gastric metaplastic cells among Japanese living in Hawaii. Jpn J Cancer Res 1991;82:86-9.

14. Rubio CA, Sugano H, Kitagawa T, Kato Y, Hirota T, Hirohashi S, et al. Geographic variations in the histology of the gastric mucosa: its significance in gastric carcinogenesis. In: Nishi M, Sugano H, Takahashi T, editors. Proceedings of the First International Gastric Cancer Congress. Bologna: Moduzzi Edittore; 1995. pp. 369-72.

15. Rubio CA, Owen D. A comparative study between the gastric mucosa of Western Canadians and other dwellers of the Pacific Basin. Antiancer Res 1998;18:2463-70.

16. De Manzoni G, Morgagni P, Roviello F, Di Leo A, Saragoni L, Marrelli D, et al. Nodal abdominal spread in adenocarcinoma of the cardia. Results of a multicenter prospective study. Gastric Cancer 1998;1:146-51.

17. Rubio CA, Kato Y, Sugano H, Kitagawa T. Intestinal metaplasia of the stomach. I. Quantitative analysis in gastric peptic ulcer and in incipient adenocarcinoma in Japanese patients. Anticancer Res 1985;5:435-40.

18. Rubio CA, Hirota $\mathrm{T}$, Itabashi $\mathrm{M}$, Hirohashi $\mathrm{S}$, Kato $\mathrm{Y}$. Quantitation of gastric intestinal metaplasia by morphometry in Japanese patients. Jpn J Cancer Res 1992;83:495-8.

19. Rivera F, Rubio CA. Quantitative studies of the extension of gastric intestinal metaplasia in gastrectomy specimens from Swedish patients. Eur J Gastroenterol Hepatol 1993;5:5215.

20. Laurén PA, Nevalainen TJ. Epidemiology of intestinal and diffuse types of gastric carcinoma. Cancer 1993;71:292633.

21. Rubio CA, Stemmermann G. Geographic variations in the phenotype of ciliated gastric cells. J Environ Pathol Toxicol Oncol 1993;12:93-9.

22. Rubio CA, Hubbard G. A new phenotype of gastric pyloric cells. A study in baboons. In Vivo. 1998;12:543-6.

23. Rubio CA, Hirota T, Itabashi M, Jacobsson B, Lignelid H. Eosinophilic bodies in pyloric and Brunner's gland cells. J Clin Pathol 1992;45:1119-20.

24. Befrits R, Granström M, Rylander M, Rubio CA. Helicobacter pylori in 205 consecutive endoscopy patients. Scand J Infect Dis 1993;25:185-91.

25. Su B, Hellström P, Rubio CA, Çelik J, Granström M, Normark S. Helicobacter pylori (type I) shows Lewis ${ }^{\mathrm{b}}$ independent adherence to gastric cells requiring de novo protein synthesis in both host and bacteria. J Infect Dis 1998;178:567-89. 